\title{
Plötzlicher, unerwarteter Tod bei Epilepsie: Mechanismen und Prävention
}

Max Christian Pensel, Rainer Surges

\author{
SUDEP ist ein Akronym aus dem Englischen (sudden, unexpected death in epilepsy) \\ und benennt den plötzlichen, unerwarteten Tod von Menschen mit Epilepsie. Es \\ handelt sich um eine seltene, aber gravierende Gefahr für Epilepsiepatienten. Im \\ Folgenden soll gezeigt werden, welche Mechanismen und Risikofaktoren eine Rolle \\ spielen und wie das Auftreten von SUDEP verhindert werden kann.
}

\section{Definition und Epidemiologie}

\author{
Merke \\ SUDEP bezeichnet den Tod von Menschen mit \\ Epilepsie, der - beobachtet oder unbeobachtet - \\ plötzlich und unerwartet auftritt und für den eine \\ Autopsie keine alternative strukturelle oder \\ toxikologische Erklärung aufzeigen kann [1].
}

Ein epileptischer Anfall kann SUDEP unmittelbar vorausgehen, stellt aber kein zwingendes Kriterium dar. Ein dokumentierter Status epilepticus über mindestens $30 \mathrm{Min}$ Dauer schließt einen SUDEP jedoch aus [1]. SUDEP verursacht wahrscheinlich zwischen 5 und $30 \%$ aller vorzeitigen Todesfälle von Epilepsiepatienten. [2] Die Inzidenz für Kinder und Jugendliche bis 17 Jahre wird derzeit auf 0,22 je 1000 Patientenjahre und für Erwachsene auf 1,2 je 1000 Patientenjahre geschätzt, was einer Gesamtinzidenz von 0,58 je 1000 Patientenjahre entspricht [3]. Als Risikofaktoren wurden identifiziert:

- fokale Epilepsien mit frühem Beginn und schlechtem Ansprechen auf die Therapie,

- das häufige Auftreten generalisierter tonisch-klonischer sowie nächtlicher Anfälle,

- männliches Geschlecht sowie

- junges Lebensalter [4-7].

Schwer behandelbare Epilepsien gehen dabei in der Regel mit einer Polypharmatherapie einher, die zeitweise auch als unabhängiger Risikofaktor für SUDEP diskutiert wurde [5]. Mangelnde Compliance bezüglich der Medikamenteneinnahme begünstigt wahrscheinlich das Auftreten von SUDEP. Der Grund für diese Annahme ist, dass in der Mehrzahl der SUDEP-Fälle unzureichende Serumspiegel der Antikonvulsiva festgestellt wurden [8, 9]. SUDEP tritt meist unbeobachtet auf - die Patienten werden in der Regel am Morgen tot in ihrem Bett aufgefunden [10]. Vereinzelte
Studien führen als schwache protektive Faktoren eine positive Anamnese für zerebrovaskuläre Erkrankungen oder Asthma Bronchiale an $[2,11]$, jedoch ist nicht auszuschlieBen, dass diese Effekte auf Stichprobenverzerrungen basieren. Abgesehen von SUDEP besteht bei Epilepsiepatienten auch ein erhöhtes Risiko, frühzeitig an Pneumonien, vaskulären Erkrankungen oder tödlichen Unfällen, z. B. durch Ertrinken, zu versterben [12,13].

Merke

Das SUDEP-Risiko ist altersabhängig: vor allem Patienten mit Epilepsie im Alter von 20 bis 40 Jahren, die etwa $50 \%$ aller SUDEP-Fälle ausmachen, haben gegenüber der Allgemeinbevölkerung ein ca. 24-fach erhöhtes Risiko für einen plötzlichen und unerwarteten Tod $[14,15]$.

\section{FALLBEISPIEL}

Ein 20-jähriger, alleinlebender Mann wird am Morgen von einem befreundeten Nachbarn leblos in seinem Bett aufgefunden. Er befindet sich in Bauchlage, das Gesicht liegt auf einem großen Kissen. Der hinzugerufene Notarzt kann nur noch den Tod feststellen. Die Eltern des Mannes berichten über eine Epilepsieerkrankung bei ihrem Sohn. Zuletzt habe er die Medikamente nur noch unregelmäßig eingenommen, da ihn die Nebenwirkungen beeinträchtigten und es ausschließlich nachts zu "großen Anfällen“ gekommen sei, die aber stets nach wenigen Minuten von selbst aufgehört hätten.

\section{Die MORTEMUS-Studie}

Der klinische Ablauf des SUDEP blieb über viele Jahrzehnte ungeklärt. Erst im Jahr 2013 charakterisierten die Auto- 
ren einer multizentrischen Studie mehrere beobachtete SUDEP- und beinahe-SUDEP- Fälle in Epilepsie-MonitoringEinheiten (Mortality in Epilepsy Monitoring Unit Study [16]). Patienten mit therapierefraktärer Epilepsie können im Rahmen eines Epilepsie-Monitorings ihre Anfälle in spezialisierten Zentren aufzeichnen lassen, um die Möglichkeit einer epilepsiechirurgischen Therapie zu überprüfen. Hierbei werden die Patienten über mehrere Tage mittels Video sowie konstanter EEG-Ableitung überwacht. Zudem werden Kreislaufparameter wie Herzfrequenz, EKG und in manchen Zentren auch die Sauerstoffsättigung des Blutes erfasst. Damit in einem absehbaren Zeitrahmen eine ausreichende Anfallsaufzeichnung gelingen kann, werden die antiepileptischen Medikamente der Patienten während des Epilepsie-Monitorings in der Regel reduziert und teilweise sogar komplett abgesetzt.

\section{Ergebnisse der MORTEMUS-Studie}

\section{Merke}

Alle der 16 beobachteten SUDEP- und 7 der 9

beobachteten beinahe-SUDEP-Fälle in der MORTEMUSStudie traten im unmittelbaren Anschluss an einen generalisierten tonisch-klonischen Anfall auf [16].

Bei 10 der in der MORTEMUS-Studie aufgezeichneten SUDEP-Fälle konnte die zeitliche Sequenz der relevanten respiratorischen und kardialen Parameter bis zum Eintritt des Todes quantifiziert werden. Hierbei fand sich zu Beginn der postiktalen Phase zunächst eine Hyperpnoe mit

Generalisierter tonisch-klonischer Anfall

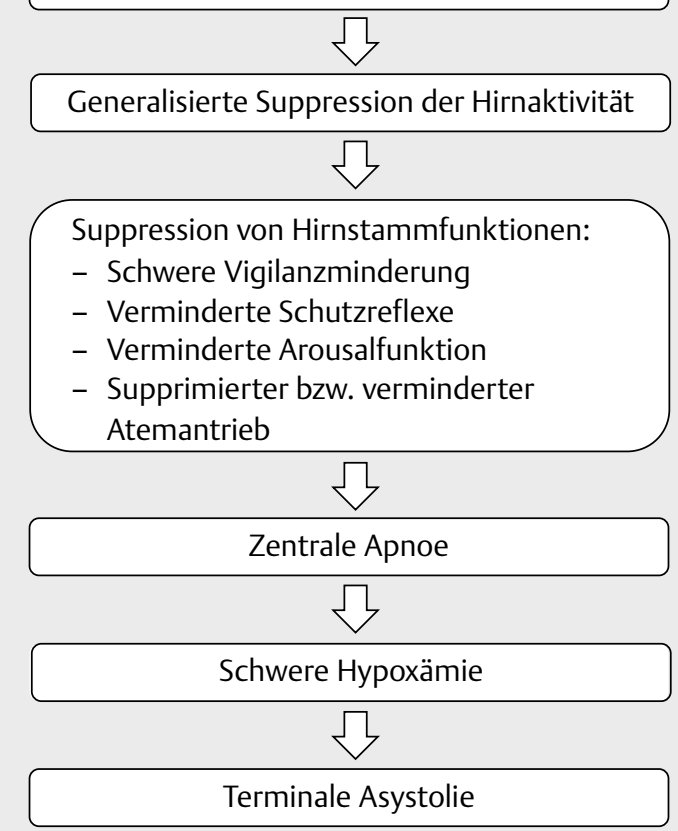

Abb. 1 Ablauf des postikalen vegetativen Zusammenbruchs (Schema).
18 bis 50 Atemzügen pro Minute, gefolgt von Apnoeperioden, die $25-180$ s postiktal einsetzten. Innerhalb der ersten drei Minuten kam es zu einer frühen kardialen Dysfunktion (15-140 s postiktal), die als Bradykardie begann und bei 9 von 10 Patienten 20-190 s postiktal in eine Asystolie mündete. Nach Anfallsende flachten die Amplituden im EEG jeweils ab - ein möglicher Ausdruck einer generalisierten Suppression der Hirnaktivität. Im zeitlichen Ablauf ereignete sich die terminale Apnoe stets vor der terminalen Asystolie. Während bei 3-4 der Patienten bereits die frühe kardiale Dysfunktion zum Tod führte, zeigten die übrigen Patienten zunächst eine kurzzeitige Erholung von Atmung und Herzfunktion. Nach wenigen Minuten trat aber auch bei ihnen ein vollständiger Kreislaufstillstand ein. In 2 von 5 aufgezeichneten beinahe-SUDEP-Fällen zeigte sich ein ähnlicher Ablauf (generalisierter Anfall gefolgt von Apnoe und Asystolie). Die Autoren der MORTEMUS-Studie bezeichneten dieses Muster als frühen postiktalen neurovegetativen Zusammenbruch („early postictal neurovegetative breakdown“), der entweder unmittelbar oder verzögert zum Tod führt ( $\triangleright$ Abb. 1). Interessant: in 2 der in der MORTEMUS-Studie beschriebenen beinahe-SUDEP-Fälle ist der postiktale neurovegetative Zusammenbruch nach fokalen Anfällen ohne sekundäre Generalisierung aufgetreten. Während tatsächlicher SUDEP meistens nachts auftrat, ist es zu beinaheSUDEP-Fällen häufiger tagsüber gekommen [16].

\section{Atmungsregulation und anfallsassoziierte respiratorische Dysfunktion}

\author{
Merke \\ Die anfallsassoziierte respiratorische Funktionsstörung \\ scheint eine zentrale Rolle beim SUDEP zu spielen. \\ Vermutlich führt die Propagation einer langsamen \\ Depolarisationswelle zum Hirnstamm während eines \\ Anfalls zu einer Dysregulation primär der Atmung und \\ sekundär der Herzaktivität führt $[10,17,18]$.
}

Eine Autopsie von an SUDEP-verstorbenen Patienten hat in $62 \%$ der Fälle pulmonale Ödeme nachgewiesen [19]. Vorübergehende zentrale Apnoen kommen bei unterschiedlichen Anfallstypen auch unabhängig von SUDEP in einer Häufigkeit von 33 bis 43 \% der Fälle vor [20, 21]. Pulmonale Ödeme nach massiver Katecholamin-Freisetzung wurden ebenfalls im Anschluss an generalisierte Anfälle beobachtet, die nicht in einen SUDEP mündeten $[10,22,23]$. Zudem wurde in einer Studie bei $33 \%$ der aufgezeichneten fokalen Anfälle ein relevanter Anstieg des endtidalen $\mathrm{CO}_{2}$-Gehalts gemessen [24]. In der MORTEMUS-Studie wurden Patienten, die an einem SUDEP verstorben sind, überdurchschnittlich häufig in Bauchlage vorgefunden, was möglicherweise die ineffektive postiktale Atmung weiter erschwerte [16]. 


\section{FALLBEISPIEL}

In der Autopsie findet sich ein Zungenbiss und ein pulmonales Ödem, was auf einen abgelaufenen generalisierten tonisch-klonischen Anfall hindeutet. Die Serumspiegel der Antiepileptika liegen deutlich unterhalb des typischen Referenzbereichs. Es ergeben sich keine Hinweise auf eine Todesursache durch Intoxikation oder Trauma. Auch findet sich kein Hinweis auf eine über die Epilepsie hinausgehende Erkrankung.

\section{Postiktale EEG-Suppression}

Bei den in der MORTEMUS-Studie aufgezeichneten SUDEP-Fällen zeigte sich postiktal eine generalisierte EEG-Suppression (PGES) mit Amplituden nicht größer als $10 \mu \mathrm{V}$ $[16,25]$. Ein derartiges EEG-Phänomen wird allerdings häufig auch nach generalisierten Anfällen beobachtet, die nicht zum Tode führen. Daher handelt es sich zunächst nicht um ein SUDEP-spezifisches Merkmal ( Abb. 2)[16]. Allerdings konnte gezeigt werden, dass es insbesondere nachts, also zu der für SUDEP typischen Zeit, nach generalisierten Anfällen häufiger zu PGES kommt [26, 27]. Es ist derzeit noch nicht abschließend geklärt, ob eine besonders lange Dauer der postiktalen EEG-Suppression als Prädiktor eines künftigen für SUDEP angesehen werden kann $[17,27,28]$.

\section{Kardiovaskuläre Dysfunktion}

\section{Merke}

Die aufgezeichneten SUDEP-Patienten der MORTEMUSStudie zeigten innerhalb der ersten 3 min postiktal eine kardiale Dysfunktion, die insbesondere durch eine sekundäre Bradykardie mit weniger als 45 Schlägen/ Minute gekennzeichnet war, bevor direkt oder nach einer kurzen Erholung eine Asystolie einsetzte [16].

In weiteren veröffentlichten SUDEP- oder beinahe-SUDEPFällen konnten als seltene aber schwerwiegende Anfallsfolge teilweise kardiale Arrhythmien festgestellt werden [29-31]. In dieser Hinsicht wird im Rahmen der SUDEP-Pathogenese eine autonome Dysfunktion diskutiert [31, 32]. Epilepsiepatienten weisen insgesamt eine erniedrigte Herzfrequenzvariabilität und somit eine verminderte vagale bzw. eine erhöhte sympathische Aktivität auf, was auf ein erhöhtes kardiovaskuläres Risiko hindeutet [32]. In einem Fall wurde bei einem SUDEP-Patienten eine verkürzte QT-Zeit beobachtet, während es bei epileptischen Anfällen im Allgemeinen häufiger zu QT-Verlängerungen kommt, vor allem bei einer Anfallsgenese im linken Temporallappen [31, 33]. Unabhängig von SUDEP sind epileptische Anfälle häufig mit iktalen Tachykardien und seltener auch mit iktalen Bradykardien und Asystolien vergesellschaftet [10, 34, 35]. Möglicherweise führen wiederholte
Anfälle dazu, dass es bei länger andauernder Erkrankung zu strukturellen Änderungen von autonomen Zentren und damit zu interiktalen Änderungen der Herzfrequenz kommt [36]. Zudem können im Zuge einer durch generalisierte Anfälle ausgelösten Katecholaminfreisetzung auch Tako-Tsubo-Kardiomyopathien entstehen [37]. Insgesamt ist das Verhältnis von kardialen Erkrankungen und Epilepsien sehr komplex und reicht über Fragen der Pathophysiologie des SUDEP weit hinaus [38].

\section{Arterieller Blutdruck}

Die Regulation des arteriellen Blutdrucks im epileptischen Anfall ist wenig untersucht. Eine klinische Studie zeigte zumindest, dass der arterielle Blutdruck regelhaft mit fokalen und generalisierten tonisch-klonischen Anfällen ansteigt und kurze Zeit nach Anfallsende wieder auf die Ausgangswerte abfällt [39]. In diesem Zusammenhang ist eine Kasuistik erwähnenswert, bei der nach einem generalisierten tonisch-klonischen Anfall eine relevante arterielle Hypotension aufgetreten ist. Diese wurde als mögliche SUDEP-Ursache diskutiert [40]. Eine vorübergehende Verminderung des Baroreflexes nach generalisierten tonisch klonischen Anfällen könnte das Auftreten einer relevanten Hypotension begünstigen [41].

\section{Risikoeinschätzung für SUDEP}

Auch wenn im Laufe der Zeit immer mehr pathophysiologische und klinische Details des SUDEP beschrieben wurden, fehlen verlässliche Biomarker zur individuellen Risikoabschätzung. Erschwerend kommt hinzu, dass SUDEP wahrscheinlich verschiedene Ursachen hat.

\section{Obwohl die überwiegende Anzahl von SUDEP-Fällen mit Anfällen bei schwer behandelbarer Epilepsie auftritt, wurde SUDEP auch in Abwesenheit von Anfällen beschrieben. Deshalb kann eine Fokussierung auf (peri-)iktale Mechanismen nicht das gesamte Spektrum von SUDEP-Fällen erklären $[42,43]$.}

\section{Die Suche nach verlässlichen Biomarkern}

Die meisten pathophysiologischen Erklärungsansätze liegen derzeit für den anfallsassoziierten SUDEP vor. So konnten auf genetischer Ebene im Tiermodell bei einer Serotoninrezeptor-Knockout-Maus (5-HT2C) epileptische Anfälle beobachtet werden, die in einen tödlichen Atemstillstand mündeten [44]. Weitere Tierexperimente zeigten in Mäusen mit bestimmten genetischen Ausstattungen (DBA/1 und DBA/2) einen Zusammenhang von verminderter Serotoninproduktion und -transmission mit tonischen Anfällen. Die Anfälle führten zunächst zu einem Atemstillstand und im Verlauf zu einer terminalen Asystolie [45-48]. Eine weitere im Tiermodell generierte Theorie sieht in einer anfallsassoziierterhöhten Adenosinkonzentration einen wichtigen Faktor, der nachfolgende Apnoen und Asystolien auslösen kann [49]. Darüber hinaus scheinen endogene Opioide eine wichtige 


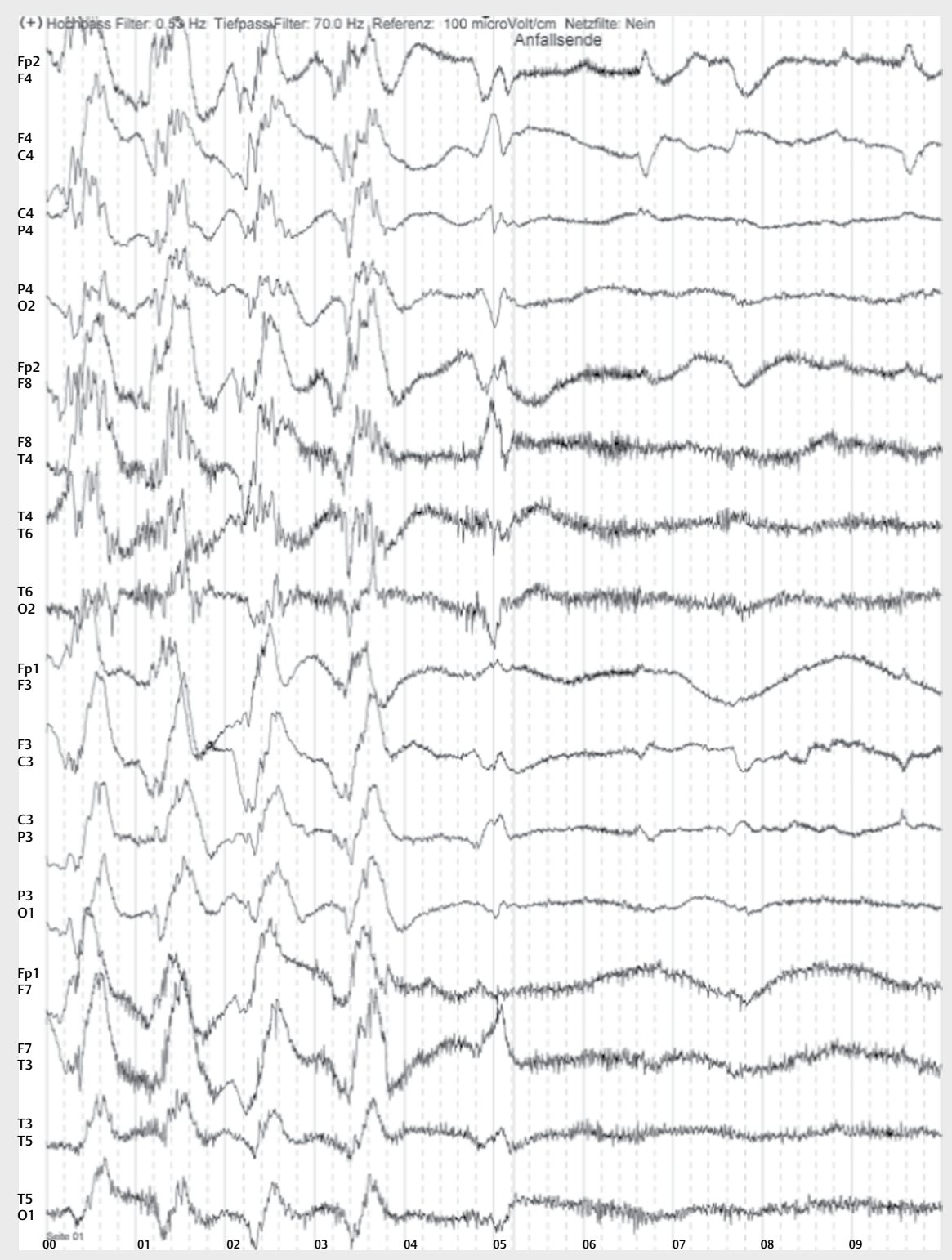

Abb. 2 Das EEG zum Ende eines generalisierten tonisch-klonischen Anfalls zeigt neben Bewegungsartefakten eine postiktale generalisierte EEG-Suppression (PGES).

Rolle bei der zentralen iktalen Aktivität zu spielen, sodass auch hier ein Zusammenhang mit der SUDEP-Entstehung diskutiert wird [50]. Aufgrund der Vergesellschaftung von epileptischen Anfällen mit teilweise tödlichen kardialen Ereignissen wurden auch genetische Loci untersucht, denen eine doppelte Rolle in Bezug auf neuronale und kardiale Funktionen zukommt [51]. In der strukturellen Neurobildgebung mittels Magnetresonanztomografie konnten ebenfalls erste Ergebnisse erzielt werden, die die Ermittlung von SUDEP-spezifischen Biomarkern in diesem Bereich in Zukunft möglich erscheinen lassen. SUDEP-assoziierte Phänomene waren z. B. eine volumetrische Zunahme der grauen Substanz in rechtsseitigen mesiotemporalen Strukturen mit gleichzeitiger Abnahme im posterioren
Thalamus sowie spezifische Atrophiemuster des Hirnstamms $[52,53]$.

\section{Vorbeugung}

\section{Anfallsreduktion als wichtigste Präventionsmaßnahme}

SUDEP tritt in den meisten Fällen wahrscheinlich nach generalisierten, tonischklonischen Anfällen auf. Bereits Patienten mit 1-2 generalisierten tonisch-klonischen Anfällen pro Jahr haben ein etwa 5-fach erhöhtes Risiko für SUDEP gegenüber Patienten ohne generalisierte tonisch-klonische 
Anfälle [54]. Daher ist die Prävention gerade dieses Anfallstyps derzeit der vielversprechendste Ansatz zur Verhinderung des SUDEP. Dies kann insbesondere durch eine adäquate antiepileptische Pharmatherapie erreicht werden, wobei der Compliance des Patienten eine wichtige Rolle zukommt [55]. Wichtige Faktoren für eine regelmäßige Einnahme der antiepileptischen Medikation sind der sozioökonomische Status und eine tolerable Belastung durch Nebenwirkungen [56]. Durch medikamentöse Therapie kann bei ca. 2/3 aller Epilepsiepatienten vollständige Anfallsfreiheit erzielt werden [57]. Bei den verbleibenden schwer behandelbaren Epilepsien kann für geeignete Kandidaten durch einen erfolgreichen epilepsiechirurgischen Eingriff das Risiko für epileptische Anfälle und für SUDEP relevant vermindert werden [58]. Zudem wurde gezeigt, dass auch eine neuromodulatorische Therapie mittels Vagusnerv-Stimulation das SUDEP-Risiko reduzieren kann [59].

\section{Weitere Möglichkeiten der Vorbeugung}

Eine vollständige Anfallsfreiheit ist bei einem Teil der Epilepsiepatienten nicht erreichbar. Daher kann in einem zweiten Schritt versucht werden, in der SUDEP-spezifischen fatalen Kaskade anzusetzen, die postiktal zunächst zu respiratorischen und dann zu kardialen Ausfällen führt. In Mausmodellen konnte bereits durch Gabe von Serotonin-Wiederaufnahme-Hemmern (SSRI) einer postiktalen zentralen Apnoe erfolgreich entgegengewirkt - die SUDEP-Rate sank. In den entsprechenden Studien wurden Mäuse der Linien DBA/1 und DBA/2 verwendet. Diese zeigten nach audiogenen Anfällen einen respiratorischen $\mathrm{Ar}$ rest zeigten, wobei der Ablauf durch serotonerge Mechanismen modulierbar war $[46,60]$. Hier könnten präventive Strategien auch beim Menschen ansetzen, bspw. im Sinne einer prophylaktischen Gabe von SSRI bei Patienten mit erhöhtem SUDEP-Risiko. Erste pharmakologische Studien zeigten eine ausreichende Sicherheit vor Nebenwirkungen und erbrachten teilweise sogar Hinweise auf ein mögliches antiepileptisches Potential dieser Substanzklasse [61-64]. Zudem konnte in einer retrospektiven Studie gezeigt werden, dass es bei Epilepsiepatienten unter SSRI-Medikation seltener zu relevanten respiratorischen Dysfunktionen nach fokalen Anfällen gekommen war als bei Patienten einer gleichaltrigen Vergleichsgruppe [65]. Eine prospektive, kontrollierte Prüfung steht jedoch noch aus.

Ein weiterer, noch weitgehend spekulativer Ansatzpunkt ist das Adenosinsystem. Dieses ist nicht nur pharmakologisch, z. B. durch Koffein als Adenosinrezeptorantagonist, sondern auch durch Lebensstilfaktoren wie Diät, Schlaf und Sport beeinflussbar. Einer möglicherweise durch Opioide mitvermittelte Atemdepression könnte im akuten Fall ggf. durch Antagonisten wie Naloxon oder Naltrexon vorgebeugt werden $[66,67]$. Daneben stellt auch die Organisation und technische Ausstattung von Epilepsie-Monitor-Einheiten einen wichtigen Ansatzpunkt dar, da die Patienten hier aufgrund der häufig stattfindenden pharmakologischen Abdosierung besonders gefährdet sind. In der MORTEMUS-
Studie zeigte sich, dass der postiktale neurovegetative Zusammenbruch im Rahmen des SUDEP innerhalb der ersten drei Minuten abläuft. Patienten, bei denen Wiederbelebungsmaßnahmen in diesem Zeitfenster begonnen wurden, konnten erfolgreich reanimiert werden [16]. Ein früher Beginn lebenserhaltender Sofortmaßnahmen stellt also eine wichtige Strategie dar, sofern es bereits zur Initiierung des neurovegetativen Zusammenbruchs gekommen ist. Da es insbesondere nachts zu SUDEP-Fällen kommt, sollte die permanente Besetzung von EEG-Monitoreinheiten mit in Wiederbelebungsmaßnahmen geschultem Personal gewährleistet sein [6].

Merke
Es konnte gezeigt werden, dass nächtliche
Überwachung auch in häuslicher Umgebung einen
positiven Beitrag zur Verhinderung von SUDEP leisten
kann, sei es durch technische Vorrichtungen oder
durch das Schlafen einer weiteren Person im Raum des
Patienten [11]. Eventuell kann zudem durch Gebrauch
eines kleinen, harten Schlafkissens (Anti-Asphyxie-
Kissen) einer postiktalen Hypoxie relevant
entgegengewirkt werden [68].

\section{Aufklärung über SUDEP}

Ein weiterer wichtiger Aspekt betrifft die Aufklärung über SUDEP als mögliche Komplikation einer Epilepsie. Das Wissen über SUDEP ist bei Epilepsiepatienten gering ausgeprägt, wie 2 kürzlich erschienene Studien übereinstimmend in einem tertiären Epilepsiezentrum in Australien (14\%) und in Deutschland (13\%) festgestellt haben $[69,70]$.

Ein großer Teil der Epilepsiepatienten und ihrer Angehörigen wünschen, über das individuelle Risiko für schwerwiegende Komplikationen wie SUDEP aufgeklärt zu werden, auch wenn die Eintrittswahrscheinlichkeit gering ist. Hierbei variieren die Studienergebnisse jedoch hinsichtlich des aufklärungswilligen Patientenanteils beträchtlich (89\% der Patienten in einem australischen gegenüber ca. $50 \%$ in einem deutschen Epilepsiezentrum [69, 70]). In Großbritannien wird seit einigen Jahren in einer offiziellen Leitlinie empfohlen, eine bedarfsgerechte Aufklärung über das individuelle SUDEP-Risiko als Teil der Beratung von Kindern, Jugendlichen und Erwachsenen mit Epilepsie sowie ihren Angehörigen und Betreuern vorzunehmen [71]. Einer Empfehlung der amerikanischen Epilepsiegesellschaft zufolge sollten Erwachsene Patienten darüber informiert werden, dass SUDEP ca. 1 von 1000 Epilepsiepatienten betrifft. Für Kinder mit Epilepsie sollte dementsprechend eine Aufklärung der Angehörigen darüber erfolgen, dass SUDEP in 1 von 4500 Kindern mit Epilepsie auftritt [3]. Um übertriebenen Ängsten entgegenzuwirken, soll in diesen $\mathrm{Zu}$ sammenhang darauf hingewiesen werden, wie viele Patienten statistisch gesehen nicht an SUDEP versterben (999 von 1000 Erwachsenen und 4499 von 4500 Kindern 
mit Epilepsie) [72, 73]. Der Empfehlung, Epilepsiepatienten und ihre Angehörigen und Betreuer über SUDEP aufzuklären, schließen sich die Autoren dieses Artikels ausdrücklich an. Hierbei ist jedoch anzumerken, dass ein individuelles Risiko anhand der bisherigen Studienlage nur abgeschätzt, jedoch nicht exakt berechnet werden kann [74].

\section{FALLBEISPIEL}

Als Todesursache wird letztlich ein SUDEP festgestellt. Die Eltern des Patienten erinnern sich nun daran, dass ihr behandelnder Neurologe sie und ihren Sohn einmal in einem Nebensatz über diese seltene Komplikation aufgeklärt hat. Aufgrund der geringen Eintrittswahrscheinlichkeit für SUDEP habe ihr Sohn sich jedoch weitgehend sicher gefühlt. Zudem habe er nach dem Auszug aus dem Elternhaus ein selbstbestimmtes Leben führen und die Epilepsieerkrankung so weit wie möglich vergessen wollen. Die Antiepileptika hätten ihn zuletzt insbesondere dabei behindert, Gewicht zu verlieren und in der Berufsschule gute Leistungen zu erbringen.

\section{KERNAUSSAGEN}

Der plötzliche, unerwartete Tod bei Epilepsie (SUDEP) stellt eine seltene, aber gravierende Gefahr für Epilepsiepatienten dar. Obwohl es sich um eine noch relativ wenig verstandene Komplikation handelt, konnte gezeigt werden, dass generalisierte tonisch-klonische Anfälle für das individuelle Risiko und häufig auch für die Pathophysiologie eine wichtige Rolle spielen.

- SUDEP-Fälle, die in Video-EEG-Einheiten aufgezeichnet werden konnten, gingen mit einer drastischen postiktalen, neurovegetativen Funktionsstörung einher, die zunächst zu einem Atemstillstand und im Verlauf zu einer Asystolie führte.

- SUDEP tritt häufig nachts auf und junge, männliche Patienten sind etwas häufiger betroffen.

- Maßnahmen zur Verhinderung von SUDEP betreffen insbesondere die meist pharmakologische Kontrolle von generalisierten tonisch-klonischen Anfällen sowie eine nächtliche Überwachung von gefährdeten Patienten.

\section{TAKE HOME MESSAGES}

- Sudden unexpected death in epilepsy is a rare but severe threat for epilepsy patients. Although still poorly understood, it was already shown that generalized tonic-clonic seizures are involved in the pathophysiology and therefore play an important role as a risk factor.

- SUDEP cases that were recorded in Video-EEGUnits came along with a postictal neurovegetative breakdown, leading to primary apnoea and finally to asystole.

- SUDEP is occurring mostly at night with young, male patients being predominantly at risk.

- Measures to prevent SUDEP include the control of generalized tonic-clonic seizures as well as nocturnal surveillance of patients at higher risk.

\section{Interessenkonflikt}

Max C. Pensel gibt an, dass keine Interessenskonflikte vorliegen. Rainer Surges hat Honorare für Vorträge oder Beratertätigkeiten von folgenden Firmen erhalten: Bial, Cyberonics, Desitin, Eisai, LivaNova, Novartis und UCB Pharma.

\section{Autorinnen/Autoren}

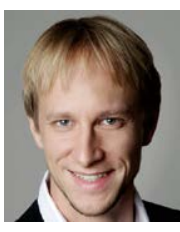

Max Christian Pensel, M.A. Jahrgang 1984. Studium der Humanmedizin, Philosophie und juristischer Teilgebiete an der Rheinischen Friedrich-Wilhelms-Universität Bonn. 2011 Magister Artium. 2013 Ärztliche Approbation. Seit 2014 Facharztausbildung Neurologie mit Schwerpunkt Epileptologie in Bonn und Aachen.

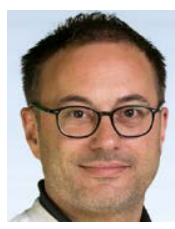

Priv.-Doz. Dr. med Rainer Surges, MHBA. Studium der Humanmedizin an der Rheinischen Friedrich-Wilhelms-Universität Bonn. Facharzt für Neurologie (Universitätskliniken Freiburg i.Br. und Bonn). Epileptologische Ausbildung am Epilepsiezentrum Freiburg, am Institute of Neurology in London sowie an der Klinik für Epileptologie in Bonn. Seit 2017 Leitung der neu gegründeten Sektion Epileptologie an der Klinik für Neurologie der Uniklinik RWTH Aachen.

\section{Korrespondenzadresse}

Priv.-Doz. Dr. med. Rainer Surges, MHBA

Neurologische Klinik, Sektion für Epileptologie Uniklinik RWTH Aachen

Pauwelsstraße 30

52074 Aachen

rsurges@ukaachen.de 


\section{Wissenschaftlich verantwortlich gemäß Zertifizierungsbestimmungen}

Wissenschaftlich verantwortlich gemäß Zertifizierungsbestimmungen für diesen Beitrag ist PD Dr. med Rainer Surges, Aachen.

\section{Literatur}

[1] Nashef L, So EL, Ryvlin P et al. Unifying the definitions of sudden unexpected death in epilepsy. Epilepsia 2012; 53: 227-233

[2] Nilsson L, Farahmand BY, Persson P-G et al. Risk factors for sudden unexpected death in epilepsy: A case control study. The Lancet 1999; 353: 888-893

[3] Harden C, Tomson T, Gloss D et al. Practice guideline summary: Sudden unexpected death in epilepsy incidence rates and risk factors: Report of the Guideline Development, Dissemination, and Implementation Subcommittee of the American Academy of Neurology and the American Epilepsy Society. Neurology 2017; 88: 1674-1680

[4] Lhatoo SD, Sander JWAS. Cause-specific mortality in epilepsy. Epilepsia 2005; 46 (Suppl 11): 36-39

[5] Hesdorffer DC, Tomson T, Benn E et al. Do antiepileptic drugs or generalized tonic-clonic seizure frequency increase SUDEP risk? A combined analysis. Epilepsia 2012; 53: 249-252

[6] Lamberts R], Thijs RD, Laffan A et al Sudden unexpected death in epilepsy: People with nocturnal seizures may be at highest risk. Epilepsia 2012; 53: 253-257

[7] Monté CPJA, Arends JBAM, Tan IY et al. Sudden unexpected death in epilepsy patients: Risk factors. A systematic review. Seizure 2007; 16: 1-7

[8] George JR, Davis GG. Comparison of anti-epileptic drug levels in different cases of sudden death. J Forensic Sci 1998; 43: 598-603

[9] Clark D, Riney K. A population-based post mortem study of sudden unexpected death in epilepsy. J Clin Neurosci 2016; 23: $58-62$

[10] Surges R, Thijs RD, Tan HL et al. Sudden unexpected death in epilepsy: risk factors and potential pathomechanisms. Nat Rev Neurol 2009; 5: 492-504

[11] Langan Y, Nashef L, Sander JW. Case-control study of SUDEP. Neurology 2005; 64: 1131-1133

[12] Neligan A, Bell GS, Johnson AL et al. The long-term risk of premature mortality in people with epilepsy. Brain 2011; 134 (Pt 2): 388-395

[13] Bell GS, Gaitatzis A, Bell CL et al. Drowning in people with epilepsy: How great is the risk? Neurology 2008; 71: 578-582

[14] Ficker DM, So EL, Shen WK et al. Population-based study of the incidence of sudden unexplained death in epilepsy. Neurology 1998; 51: 1270-1274

[15] Thurman DJ, Hesdorffer DC, French JA. Sudden unexpected death in epilepsy: Assessing the public health burden. Epilepsia 2014; 55: 1479-1485

[16] Ryvlin P, Nashef L, Lhatoo SD et al. Incidence and mechanisms of cardiorespiratory arrests in epilepsy monitoring units (MORTEMUS): A retrospective study. The Lancet Neurology 2013; 12: 966-977

[17] Hirsch LJ. Is sudden unexpected death in epilepsy due to postictal brain shutdown? Ann Neurol 2010; 68: 773-775
[18] Aiba I, Noebels JL. Spreading depolarization in the brainstem mediates sudden cardiorespiratory arrest in mouse SUDEP models. Sci Transl Med 2015; 7: 282ra46

[19] Kloster R, Engelskjøn T. Sudden unexpected death in epilepsy (SUDEP): A clinical perspective and a search for risk factors. J Neurol Neurosurg Psychiatry 1999; 67: 439-444

[20] Bateman LM, Li C-S, Seyal M. Ictal hypoxemia in localizationrelated epilepsy: Analysis of incidence, severity and risk factors. Brain 2008; 131 (Pt 12): 3239-3245

[21] Nashef L, Walker F, Allen P et al. Apnoea and bradycardia during epileptic seizures: Relation to sudden death in epilepsy. J Neurol Neurosurg Psychiatry 1996; 60: 297-300

[22] Darnell JC, Jay S]. Recurrent postictal pulmonary edema: A case report and review of the literature. Epilepsia 1982; 23: 71-83

[23] Romero Osorio OM, Abaunza Camacho JF, Sandoval Briceño $D$ et al. Postictal neurogenic pulmonary edema: Case report and brief literature review. Epilepsy Behav Case Rep 2018; 9: 49-50

[24] Seyal M, Bateman LM, Albertson TE et al. Respiratory changes with seizures in localization-related epilepsy: Analysis of periictal hypercapnia and airflow patterns. Epilepsia 2010; 51: 1359-1364

[25] Tomson T, Nashef L, Ryvlin P. Sudden unexpected death in epilepsy: Current knowledge and future directions. Lancet Neurol 2008; 7: 1021-1031

[26] Lamberts RJ, Laranjo S, Kalitzin SN et al. Postictal generalized EEG suppression is not associated with periictal cardiac autonomic instability in people with convulsive seizures. Epilepsia 2013; 54: 523-529

[27] Peng W, Danison JL, Seyal M. Postictal generalized EEG suppression and respiratory dysfunction following generalized tonic-clonic seizures in sleep and wakefulness. Epilepsia 2017; 58: 1409-1414

[28] Lamberts RJ, Gaitatzis A, Sander JW et al. Postictal generalized EEG suppression: An inconsistent finding in people with multiple seizures. Neurology 2013; 81: 1252-1256

[29] Dasheiff RM, Dickinson LJ. Sudden unexpected death of epileptic patient due to cardiac arrhythmia after seizure. Arch Neurol 1986; 43: 194-196

[30] Espinosa PS, Lee JW, Tedrow UB et al. Sudden unexpected near death in epilepsy: Malignant arrhythmia from a partial seizure. Neurology 2009; 72: 1702-1703

[31] Jeppesen J, Fuglsang-Frederiksen A, Brugada R et al. Heart rate variability analysis indicates preictal parasympathetic overdrive preceding seizure-induced cardiac dysrhythmias leading to sudden unexpected death in a patient with epilepsy. Epilepsia 2014; 55: e67-e71

[32] Lotufo PA, Valiengo L, Benseñor IM et al. A systematic review and meta-analysis of heart rate variability in epilepsy and antiepileptic drugs. Epilepsia 2012; 53: 272-282

[33] Surges R, Jordan A, Elger CE. Ictal modulation of cardiac repolarization, but not of heart rate, is lateralized in mesial temporal lobe epilepsy. PLoS ONE 2013; 8: e64765

[34] Duplyakov D, Golovina G, Lyukshina N et al. Syncope, seizure-induced bradycardia and asystole: Two cases and review of clinical and pathophysiological features. Seizure 2014; 23: 506-511

[35] Fava I, Del Gaudio S, Volpe E et al. The ictal bradycardia syndrome: A case report. Epilepsy Behav Case Rep 2015; 4: 9-12

[36] Wasterlain CG, Fujikawa DG, Penix L et al. Pathophysiological mechanisms of brain damage from status epilepticus. Epilepsia 1993; 34 (Suppl 1): S37-S53 
[37] Stöllberger C, Wegner C, Finsterer J. Seizure-associated Takotsubo cardiomyopathy. Epilepsia 2011; 52: e160-e167

[38] Shmuely S, van der Lende M, Lamberts R] et al. The heart of epilepsy: Current views and future concepts. Seizure 2017; 44: $176-183$

[39] Hampel KG, Jahanbekam A, Elger CE et al. Seizure-related modulation of systemic arterial blood pressure in focal epilepsy. Epilepsia 2016; 57: 1709-1718

[40] Bozorgi A, Chung S, Kaffashi F et al. Significant postictal hypotension: Expanding the spectrum of seizure-induced autonomic dysregulation. Epilepsia 2013; 54: e127-e130

[41] Hampel KG, Elger CE, Surges R. Impaired Baroreflex Sensitivity after Bilateral Convulsive Seizures in Patients with Focal Epilepsy. Front. Neurol 2017; 8: 210

[42] Jehi L, Najm IM. Sudden unexpected death in epilepsy: Impact, mechanisms, and prevention. Cleve Clin J Med 2008; 75 (Suppl 2): S66-S70

[43] Lhatoo SD, Nei M, Raghavan M et al. Nonseizure SUDEP: Sudden unexpected death in epilepsy without preceding epileptic seizures. Epilepsia 2016; 57: 1161-1168

[44] Tecott LH, Sun LM, Akana SF et al. Eating disorder and epilepsy in mice lacking $5-\mathrm{HT} 2 \mathrm{c}$ serotonin receptors. Nature 1995; 374: 542-546

[45] Venit EL, Shepard BD, Seyfried TN. Oxygenation prevents sudden death in seizure-prone mice. Epilepsia 2004; 45: 993-996

[46] Tupal S, Faingold CL. Evidence supporting a role of serotonin in modulation of sudden death induced by seizures in DBA/2 mice. Epilepsia 2006; 47: 21-26

[47] Faingold CL, Randall M, Tupal S. DBA/1 mice exhibit chronic susceptibility to audiogenic seizures followed by sudden death associated with respiratory arrest. Epilepsy Behav 2010; 17: 436-440

[48] Uteshev VV, Tupal S, Mhaskar Y et al. Abnormal serotonin receptor expression in DBA/2 mice associated with susceptibility to sudden death due to respiratory arrest. Epilepsy Res 2010; 88: 183-188

[49] Shen H-Y, Li T, Boison D. A novel mouse model for sudden unexpected death in epilepsy (SUDEP): Role of impaired adenosine clearance. Epilepsia 2010; 51: 465-468

[50] Ryvlin P, Nashef L, Tomson T. Prevention of sudden unexpected death in epilepsy: A realistic goal? Epilepsia 2013; 54: (Suppl 2): 23-28

[51] Glasscock E. Genomic biomarkers of SUDEP in brain and heart. Epilepsy Behav 2014; 38: 172-179

[52] Wandschneider B, Koepp M, Scott C et al. Structural imaging biomarkers of sudden unexpected death in epilepsy. Brain 2015; 138 (Pt 10): 2907-2919

[53] Mueller SG, Bateman LM, Laxer KD. Evidence for brainstem network disruption in temporal lobe epilepsy and sudden unexplained death in epilepsy. Neuroimage Clin 2014; 5 : 208-216

[54] Hesdorffer DC, Tomson T, Benn E et al. Combined analysis of risk factors for SUDEP. Epilepsia 2011; 52: 1150-1159

[55] Hughes JR. A review of sudden unexpected death in epilepsy: Prediction of patients at risk. Epilepsy Behav 2009; 14: 280-287

[56] Smith AW, Mara CA, Modi AC. Adherence to antiepileptic drugs in adolescents with epilepsy. Epilepsy Behav 2018; 80: 307-311

[57] Kwan P, Brodie M]. Early identification of refractory epilepsy. N Engl J Med 2000; 342: 314-319
[58] Seymour N, Granbichler CA, Polkey CE et al. Mortality after temporal lobe epilepsy surgery. Epilepsia 2012; 53: 267-271

[59] Ryvlin P, So EL, Gordon CM et al. Long-term surveillance of SUDEP in drug-resistant epilepsy patients treated with VNS therapy. Epilepsia 2018; 59: 562-572

[60] Faingold CL, Randall M, Zeng C et al. Serotonergic agents act on 5-HT3 receptors in the brain to block seizure-induced respiratory arrest in the DBA/ 1 mouse model of SUDEP. Epilepsy Behav 2016; 64 (Pt A): 166-170

[61] Kanner AM, Kozak AM, Frey M. The Use of Sertraline in Patients with Epilepsy: Is It Safe? Epilepsy Behav 2000; 1: 100-105

[62] Thomé-Souza MS, Kuczynski E, Valente KD. Sertraline and fluoxetine: Safe treatments for children and adolescents with epilepsy and depression. Epilepsy Behav 2007; 10: 417-425

[63] Favale E, Rubino V, Mainardi P et al. Anticonvulsant effect of fluoxetine in humans. Neurology 1995; 45: 1926-1927

[64] Specchio LM, ludice A, Specchio $\mathrm{N}$ et al. Citalopram as treatment of depression in patients with epilepsy. Clin Neuropharmacol 2004; 27: 133-136

[65] Bateman LM, Li C-S, Lin T-C et al. Serotonin reuptake inhibitors are associated with reduced severity of ictal hypoxemia in medically refractory partial epilepsy. Epilepsia 2010; 51: 2211-2214

[66] Richerson GB, Boison D, Faingold CL et al. From unwitnessed fatality to witnessed rescue: Pharmacologic intervention in sudden unexpected death in epilepsy. Epilepsia 2016; 57: (Suppl 1): 35-45

[67] Boison D, Sandau US, Ruskin DN et al. Homeostatic control of brain function - new approaches to understand epileptogenesis. Front Cell Neurosci 2013; 7: 109

[68] Catcheside PG, Mohtar AA, Reynolds KJ. Airflow resistance and $\mathrm{CO} 2$ rebreathing properties of anti-asphyxia pillows designed for epilepsy. Seizure 2014; 23: 462-467

[69] Xu Z, Ayyappan S, Seneviratne U. Sudden unexpected death in epilepsy (SUDEP): What do patients think? Epilepsy Behav 2015; 42: 29-34

[70] Surges R, Wrede Rv, Porschen T et al. Knowledge of sudden unexpected death in epilepsy (SUDEP) among 372 patients attending a German tertiary epilepsy center. Epilepsy Behav 2018; 80: 360-364

[71] Royal College of Physicians (UK). The Epilepsies: The Diagnosis and Management of the Epilepsies in Adults and Children in Primary and Secondary Care: Pharmacological Update of Clinical Guideline 20. London: 2012

[72] Knapp P, Raynor DK, Berry DC. Comparison of two methods of presenting risk information to patients about the side effects of medicines. Qual Saf Health Care 2004; 13: $176-180$

[73] Kahneman D, Tversky A. Choices, values, and frames. American Psychologist 1984; 39: 341-350

[74] Shankar R, Cox D, Jalihal V et al. Sudden unexpected death in epilepsy (SUDEP): development of a safety checklist. Seizure 2013; 22: 812-817

Bibliografie

DOI https://doi.org/10.1055/a-0626-6263

Klin Neurophysiol 2018; 49: 133-142

(c) Georg Thieme Verlag KG Stuttgart · New York

ISSN 1434-0275 


\section{Punkte sammeln auf CME.thiemeode}

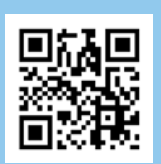

Diese Fortbildungseinheit ist 12 Monate online für die Teilnahme verfügbar.

Sollten Sie Fragen zu Online-Teilnahme haben, fi nden Sie unter cme.thieme.de/hilfe

eine ausführliche Anleitung. Wir wünschen viel Erfolg beim Beantworten

der Fragen!

Unter https://eref.thieme.de/CXAYGNY oder über den QR-Code kommen Sie direkt zum Artikel zur Eingabe der Antworten.

VNR 2760512018154653455

\section{Frage 1}

Welche Aussage zum SUDEP ist richtig?

A Epilepsiepatienten mit 1-2 generalisierten tonischklonischen Anfällen pro Jahr haben kein erhöhtes Risiko für SUDEP.

B Epilepsiepatienten mit 1-2 generalisierten tonischklonischen Anfällen pro Jahr haben ein etwa 5-fach erhöhtes Risiko für SUDEP gegenüber Patienten ohne generalisierte tonisch-klonische Anfälle.

C Das Auftreten generalisierter tonisch-klonischer Anfälle stellt keinen relevanten Risikofaktor für SUDEP dar.

D Patienten, die niemals einen generalisierten tonischklonischen Anfall erlitten haben können per Definition nicht an einem SUDEP versterben.

E SUDEP steht für „sudden unobserved death in epilepsy“.

\section{Frage 2}

Welche Aussage zum SUDEP ist falsch?

A Der SUDEP ereignet sich oft unbeobachtet.

B Der SUDEP ereignet sich oft nachts.

C SUDEP ist meist die Folge einer von der Epilepsie unabhängigen Pathologie oder Intoxikation.

D Ein epileptischer Anfall kann einem SUDEP direkt vorausgehen.

E Ein letal verlaufender Status epilepticus schließt einen SUDEP per Definition aus.

\section{Frage 3}

Welche Aussage zu Risikofaktoren des SUDEP ist richtig?

A SUDEP wird im Allgemeinen häufiger bei Kindern beobachtet als bei Erwachsenen.

B Weibliches Geschlecht und das katamenial gebundene Auftreten epileptischer Anfälle sind etablierte Risikofaktoren des SUDEP.

C Der Beginn einer Epilepsie in höherem Lebensalter ist mit einem erhöhten SUDEP-Risiko assoziiert.

D Männliches Geschlecht und das Vorliegen einer schwer behandelbaren Epilepsie sind relevante Risikofaktoren.

E Das Auftreten generalisierter tonisch-klonischer Anfälle ist ein vernachlässigbarer Risikofaktor.

\section{Frage 4}

Welche Aussage zur Prävention des SUDEP ist falsch?

A Epilepsiechirurgische Eingriffe oder die Vagusnervstimulation können das SUDEP-Risiko reduzieren.

B Die Wirksamkeit einer antikonvulsiven Medikation hat keinen Einfluss auf das SUDEP-Risiko.

C Eine rasch einsetzende kardiopulmonale Reanimation bei postiktalem Stillstand von Atmung und Herztätigkeit kann einen SUDEP verhindern.

D Die nächtliche Überwachung von Epilepsiepatienten beeinflusst das SUDEP-Risiko.

E Die Untersuchung der Wirksamkeit von Opiat-Antagonisten zur SUDEP-Prävention beim Menschen ist derzeit Gegenstand wissenschaftlicher Forschung.

\section{Frage 5}

Welche Aussage zu generalisierten tonisch-klonischen Anfällen ist falsch?

A Neurovegetative Funktionsstörungen nach generalisierten tonisch-klonischen Anfällen sind die häufigste Ursache für die meisten der bisher beschriebenen SUDEP-Fälle.

B Nach generalisierten tonisch-klonischen Anfällen kommt es häufig zu einer Amplitudenminderung im EEG.

C Ein pulmonales Ödem ist eine mögliche Komplikation generalisierter tonisch-klonischer Anfälle.

D Der arterielle Blutdruck wird durch einen generalisierten tonisch-klonischen Anfall nicht beeinflusst.

E Während epileptischen Anfällen kann es zur Zunahme der Herzfrequenz kommen. 


\section{CME-Fragen bei CMIE.thieme.de}

Fortsetzung $\ldots$

\section{Frage 6}

Welche Aussage zu Antiepileptika ist falsch?

A Belastende Nebenwirkungen haben keinen Einfluss auf die regelmäßige Einnahme der Antiepileptika durch die Patienten.

B Ziel einer antiepileptischen Therapie ist eine optimale antiepileptische Wirkung mit möglichst wenig belastenden Nebenwirkungen.

C Antiepileptika werden in speziellen Epilepsiezentren zeitweise kontrolliert abgesetzt, um epileptische Anfälle mittels kontinuierlicher Video- und EEG-Überwachung aufzuzeichnen.

D SSRI werden derzeit hinsichtlich eines möglichen antiepileptischen Potentials wissenschaftlich untersucht.

E Bei einem Anteil von ca. 2/3 aller Epilepsiepatienten kann durch antiepileptische Medikation länger andauernde Anfallsfreiheit erzielt werden.

\section{Frage 7}

Welche Aussage zur Aufklärung über SUDEP ist richtig?

A Ein überwiegender Teil der Patienten mit Epilepsie und ihrer Angehörigen ist über das Risiko eines SUDEP aufgeklärt.

B Ein Gespräch über SUDEP sollte möglichst vermieden werden, um keine unnötigen Ängste bei den Patienten und ihren Angehörigen hervorzurufen.

C Eine SUDEP-Aufklärung wird von Epilepsiepatienten und ihren Angehörigen ganz überwiegend nicht gewünscht.

D Die Einschätzung des exakten individuellen SUDEPRisikos ist anhand vorliegender Daten sehr einfach möglich.

E Gemäß einer Richtlinie aus Großbritannien sollte eine bedarfsgerechte Aufklärung über das individuelle relative SUDEP-Risiko Teil der Beratung von Kindern, Jugendlichen und Erwachsenen mit Epilepsie und ihren Angehörigen bzw. Betreuern sein.

\section{Frage 8}

Welche Aussage ist richtig?

A SUDEP macht wahrscheinlich zwischen 5 und $30 \%$ aller vorzeitigen Todesfälle von Epilepsiepatienten aus.

B Epileptische Anfälle führen nicht zu transitorischen zentralen Apnoen.

C Generalisierte tonisch-klonische Anfälle bewirken keine relevante Katecholaminfreisetzung.

D Epilepsiepatienten weisen insgesamt eine erhöhte Herzfrequenzvariabilität auf.

E Bei bisher aufgezeichneten SUDEP-Fällen ereignete sich eine terminale Apnoe stets nach einer terminalen Asystolie.

\section{Frage 9}

Welche Aussage ist falsch?

A In einem SUDEP-Tiermodell haben selektive Serotonin-Wiederaufnahmehemmer die SUDEP-Rate reduziert.

B Eine anfallsassoziierte exzessive Katecholaminfreisetzung kann ein neurogenes Lungenödem verursachen.

C Eine anfallsassoziierte exzessive Katecholaminfreisetzung kann eine Tako-Tsubo-Kardiomyopathie verursachen.

D Schlafen auf einem großen, weichen Kissen verringert das SUDEP-Risiko.

E Ein großer Anteil der an SUDEP verstorbenen Patienten wird in Bauchlage aufgefunden.

\section{Frage 10}

Welche Aussage zur Mortalität bei Epilepsie ist richtig?

A Das Risiko zu ertrinken ist bei Epilepsie nicht erhöht.

B Epilepsiepatienten im Alter von 20 und 40 Jahren haben im Vergleich zur Allgemeinbevölkerung ein ca. 24-fach erhöhtes Risiko, plötzlich und unerwartet zu versterben.

C Epilepsiepatienten um Alter zwischen 20 und 40 Jahren haben im Vergleich zur Allgemeinbevölkerung ein ca. 2,4-fach erhöhtes Risiko, plötzlich und unerwartet zu versterben

D Epilepsiepatienten haben kein erhöhtes Risiko an einer Pneumonie zu versterben.

E Epilepsiepatienten haben insgesamt kein erhöhtes Risiko vorzeitig zu versterben. 